\title{
La calidad del cuidado como desafío de las políticas públicas de atención a la dependencia
}

The quality of care as a challenge for public policies addressed at the elderly and disabled people

\section{Erika Van Rompaey}

Doctora en Sociología, Universidad Autónoma de Barcelona.

Becaria posdoctoral

CONICET - FLACSO (Argentina). evrgiuria@gmail.com
Fecha de recepción:

22.12.17

Fecha de aceptación:

19.6.18

\section{Resumen}

El artículo tiene como propósito examinar el concepto de calidad del cuidado de las personas en situación de dependencia en domicilio y sus distintos abordajes, para reflexionar sobre el impacto que tienen las condiciones materiales y técnicas de la atención ofrecida por el Estado en la protección del derecho a ser cuidado. Asimismo, analiza la calidad del cuidado provisto por el Estado en el caso uruguayo, el cual ha diseñado e implementado una nueva política de cuidados (Sistema Nacional Integrado de Cuidados) que cuenta con una prestación orientada específicamente hacia los cuidados en domicilio. El estudio del caso uruguayo se basa en una estrategia metodológica de carácter cualitativo, centrada fundamentalmente en el análisis de la documentación institucional.

Palabras clave: cuidado - dependencia - calidad políticas públicas - Uruguay.

\section{Abstract}

The purpose of the article is to examine the concept of quality of home care provided for disabled and elderly people and their different approaches, to analyse the impact that the material and technical conditions of the care offered by the State have on the protection of the right to 
be cared. Likewise, it analyzes the quality of public care in the Uruguayan case, which has designed and implemented a new care policy (National Integrated Care System) that has a program specifically aimed at home care. We select a qualitative strategy for the study of the uruguayan case, based on the analysis of institutional documents.

Key words: care - dependence - quality - public policies - Uruguay.

\section{Introducción}

La incorporación masiva de las mujeres al mercado de trabajo, el aumento de la esperanza de vida, el envejecimiento de la población y los cambios en las configuraciones familiares a partir de los setentas, plantean un nuevo problema social: cómo se resuelven los cuidados de las personas en situación de dependencia y quiénes asumen la responsabilidad al respecto. El término dependencia se utiliza para definir la condición de las personas que tienen una pérdida o ausencia de autonomía para ejecutar las actividades elementales de la vida cotidiana. Esta definición se encuentra en línea con lo sostenido por el Consejo de Europa que describe a la dependencia como "aquel estado en el que se encuentran personas que, por razones ligadas a la falta o pérdida de la autonomía fisica, psíquica o intelectual, tienen necesidad de asistencia y/o ayudas importantes para realizar las actividades de la vida diaria”. Según Torres Pérez (2005), la dependencia puede afectar a cualquier sector de población y a cualquier edad, pero fundamentalmente a las personas de edad avanzada, así como a quienes tienen discapacidades físicas, psíquicas, patologías mentales o déficits sensoriales. Así, cuando se habla de personas en situación de dependencia se hace alusión a aquellas que tienen algún tipo de discapacidad funcional, que merma o impide la capacidad de realizar por sí solas las actividades (básicas y o instrumentales) de la vida. Por ello, no todas las personas con discapacidad presentan una discapacidad funcional, es decir, se encuentran en situación de dependencia, dado que muchas de ellas no requieren de la ayuda de otra persona para llevar a cabo las actividades cotidianas de la vida.

El aumento de las personas que requieren de cuidados a la par que la disminución de la disponibilidad de las mujeres que otrora cuidaban de forma no remunerada, han incidido en lo que se ha dado a llamar como «crisis del cuidado» (Bazo, 1998; Durán 2006; Escudero 2007).

Estos cambios han impactado también en una gran proporción de las economías modernas donde se vislumbra la retracción de la industria manufacturera y el incremento 
del sector productor de servicios (Belt, Richardson y Webster 2002; Hampson y Juno 2005). Es la industria de servicios la que emplea a la mayor parte de la población ocupada a nivel mundial (Korczynski y Macdonald 2009). Es un sector que incluye un amplio y variado rango de ocupaciones, desde aquellas vinculadas a los cuidados hasta la hostelería, las finanzas, la producción y desarrollo de software, la educación, entre otros. Asimismo, el sector de servicios en general, y el de los servicios personales en particular -entre los que se encuentra los cuidados-, se caracteriza por la fuerte feminización de su fuerza laboral y la desvalorización social de sus ocupaciones (Thompson, Warhurst y Callaghan 2000; Korczynski 2002; Rigby y Sanchis 2006), las cuales suelen ser mal remuneradas (Lloyd y Payne 2002; Payne 2009).

A raíz del escenario antes mencionado se ha multiplicado la creación de los trabajos remunerados de cuidados (formales e informales en instituciones o domicilios), encarado por personas fuera del ámbito familiar. A su vez, han surgido empresas y organizaciones de la sociedad civil que ofrecen servicios -tanto públicos como privados- de cuidados, orientados a satisfacer las necesidades específicas de las personas en situación de dependencia antes cubiertas por las mujeres en el interior de los hogares de forma no remunerada. No obstante, la calidad del cuidado provisto en el marco de diversas configuraciones políticoinstitucionales, así como organizacionales de carácter público y privado y, en particular, de aquellos provistos bajo la órbita de prestaciones estatales que apuntan a garantizar el derecho a ser cuidado de sus ciudadanos, ha sido escasamente estudiado en el ámbito latinoamericano. Ello debido, en parte, a que el tema de la atención a la dependencia y sus cuidados se ha colocado recientemente como un asunto de interés público en la agenda de los gobiernos y pocos países han implementado políticas orientadas a esta población.

El artículo tiene como propósito examinar el concepto de calidad y sus distintas aproximaciones conceptuales, para reflexionar sobre el impacto que tienen las condiciones materiales y técnicas de los cuidados en domicilio ofrecidos por el Estado en la protección del derecho a ser cuidado de las personas en situación de dependencia. Para analizar el caso uruguayo se ha seleccionado una estrategia metodológica de carácter cualitativo, centrada fundamentalmente en el análisis de la documentación institucional.

\section{Las políticas de atención a la dependencia, dimensiones de análisis y la calidad de los cuidados de larga duración}

Las políticas públicas de atención a las personas en situación de dependencia -que requieren de la ayuda de otra persona para realizar las actividades de la vida diaria- pueden asumir diversas formas, de acuerdo a los enfoques conceptuales en los que se sustentan, así como en función de las capacidades, recursos y compromisos políticos de los gobiernos y Estados que las llevan a cabo. En esta línea, las políticas pueden presentar diversos diseños institucionales, con mayor o menor integración de los componentes sanitarios 
o sociales y con distintos grados de cobertura y alcance. Asimismo, pueden incluir variadas prestaciones (servicios y/o transferencias monetarias) orientadas a cubrir distintas necesidades que las personas en esta situación tienen tales como las pensiones para la subsistencia económica o material, los cuidados y soporte para la realización de las actividades diarias de la vida cotidiana o de aquellas de tipo instrumental (gestión de dinero, preparación de alimentos, uso del sistema de transporte, recreación, etc.) en su domicilio o en instituciones, así como las ayudas para el reacondicionamiento y adaptabilidad de hogares y la movilización, los sistemas de educación y de salud inclusivos, entre otras.

Varios autores han examinado las dimensiones a ser incorporadas en el análisis de las políticas públicas de atención a la población en situación de dependencia. Sin ánimo de ser exhaustivos en esta materia, dado que no es el objetivo de este artículo, y a partir de la revisión de la literatura especializada en castellano, se presentan algunas de las características centrales utilizadas para caracterizar a este tipo de política pública. En sintonía con ello, López Casasnovas (2011) propone un conjunto de dimensiones de análisis entre las que destaca el tipo de regulación (con aseguramiento obligatorio público o privado o ambos, o privado incentivado), de suministro (público directo o público privado) y el grado en que respeta o no las preferencias de las personas a recibir atención en sus propios hogares, siguiendo la tendencia internacional basada en el empoderamiento y la autodeterminación de esta población. A su vez, sostiene que otra de las dimensiones a ser tenidas en cuenta consiste en los grados de coordinación e integración interadministrativa sobre todo entre el ámbito social y sanitario tanto a nivel de la financiación y en los aspectos organizativos, de gestión e institucionales (departamento/s responsable/s). Por otro lado, Rodríguez Cabrero (2007), plantea que entre los aspectos a ser considerados en el análisis de las políticas públicas de atención a la dependencia se destacan sus grados de universalización, suficiencia, calidad y sostenibilidad. En particular, plantea que el desarrollo y garantía de los derechos sociales implica que los Estados provean de ayuda específica para fomentar la autonomía de esta población, garantizando la universalidad e intensidad protectora. Mediante la accesibilidad se establecen los límites de acceso al sistema, la cual viene marcada por los criterios del baremo que sean establecidos para el ingreso al sistema de prestaciones. Por otra parte, sostiene que la intensidad protectora depende del peso que el sistema conceda a las prestaciones orientadas a promover la autonomía personal en situación de gran dependencia frente a la dimensión paliativa o rehabilitadora.

En sintonía con lo que propone Rodríguez Cabrero, así como también lo plantea Leichsenring (2004) y López Casasnovas (2011) consideramos que, en cuanto a las diversas prestaciones que la política pública de atención a la dependencia puede ofrecer, la provisión de alternativas de cuidado de larga duración y, en particular de aquellos provisto en los domicilios, constituyen una prestación clave para el desarrollo de las actividades tanto básicas como instrumentales de la vida diaria de la población en esta situación al promover el respeto por las preferencias y la autonomía, así como garantizando la protección del derecho a ser cuidado. 
Asimismo, entre dichas prestaciones es posible distinguir varios tipos de necesidades a ser cubiertas. Por un lado, las necesidades de cuidados y soporte para realizar las actividades básicas de la vida diaria (higiene, alimentación, vestimenta, movilización dentro y fuera del hogar, control de medicación); por el otro, las de apoyo para llevar a cabo actividades instrumentales que habiliten la integración de las personas en la sociedad, tales como acceder al sistema de transporte, público, gestionar dinero y realizar actividades recreativas y comunitarias.

Desde nuestro punto de vista, el análisis de la dimensión relativa a la calidad de los cuidados de larga duración y en especial de aquellos provistos en el domicilio de las personas es un tema pendiente de ser indagado en América Latina. Por ello nos centramos en el estudio de la calidad y su significado en el ámbito de los servicios sociales y de los cuidados en domicilio, problematizando acerca de sus implicancias para la efectiva protección del derecho de las personas en situación de dependencia a ser cuidadas y vivir una vida lo más autónoma posible en sus hogares.

Una de las primeras cuestiones a analizar cuando abordamos el tema de la calidad y, en particular, de los servicios sociales y de cuidados, tiene que ver con rastrear el origen del concepto y cómo éste utilizado primero en el sector industrial, para luego aplicarse al de los servicios personales en nuestras modernas economías. Según Senlles y Gutiérrez (2005), el interés por el tema de la calidad emerge en la década de 1930 en Estados Unidos, al introducirse la estadística en la inspección de los productos acabados. Más tarde surgen aportaciones que modifican el concepto y se incluyen parámetros clave de los procesos de producción que permiten, dentro de ciertos límites, cumplir con las especificaciones de los productos. En esta línea, Japón ocupó un lugar relevante en la evolución del enfoque de la calidad, al desarrollar principios propios tales como la ventaja competitiva para los productos e involucrar a las personas en los proceso de mejora, aplicando la gestión de la calidad total a finales de los sesenta y principios de los setenta. Asimismo, sobre la base de normas militares creadas en los años sesenta, la British Standard Institution estableció parámetros básicos de la calidad que más adelante fueron adaptados por la International Organization for Standardization, siendo reagrupados en diferentes series temáticas. En 1985, la Comunidad Económica Europea (actual UE), puso de manifiesto la necesidad de aproximaciones técnicas en las distintas empresas europeas e instó a buscar una normativa que asegurara la conformidad de servicios, productos, sistemas y procesos. Así surge la Norma ISO 9000, la cual ha sufrido distintas revisiones a lo largo de los años.

Más allá de que el interés por el tema de la calidad estuvo ligado originariamente a los procesos productivos de tipo industrial, su uso fue extendido a la industria de los servicios y posteriormente a los servicios sociales. Para Senlles y Gutiérrez (2005), el enfoque de calidad aplicado a la mejora de los procesos de producción de servicios sociales tales como los educativos significa incorporar sistemas de gestión que permitan simplificar procesos, eliminar fallos, errores y costos añadidos, terminar con la no-calidad en el sentido más amplio, y especialmente, ser eficaz y eficiente. 
En sintonía con lo anterior, diversas investigaciones académicas de origen fundamentalmente anglosajón se centraron, a partir de los noventas, en el estudio de la calidad de los servicios de cuidado y, específicamente, en aquellos llevados a cabo en el domicilio de las personas adultas mayores. De acuerdo con Applebaum y Phillips (1990), los sistemas de servicios de cuidado de larga duración realizaron importantes esfuerzos durante la década de los ochentas para garantizar la calidad del cuidado provisto, en el contexto de restricciones presupuestarias y de la fuerte presión por mantener los costos lo más bajo posible derivados de las políticas neoliberales adoptadas y su impronta por la reducción o minimización del gasto del Estado y, en especial, de su gasto social. Según Malley y Fernández (2010), el estudio de la calidad de los servicios de cuidados en el mundo fue motivado por la necesidad de diversificar y tercerizar la provisión de los mismos por parte de los gobiernos, en el marco de procesos de reducción de gastos del Estado y la consecuente precarización laboral de la fuerza de trabajo en el sector de servicios. Al respecto, plantean que la separación resultante entre los responsables y proveedores de servicios, ha llevado a problemas de información, sobre todo, en relación a la habilidad de los primeros para evaluar la calidad de las prestaciones ofrecidas. Applebaum y Phillips (1990), sostienen que, si bien la relación entre la calidad y el costo de los servicios es compleja y difícil de estimar, las estrategias de reducción de costos suelen desestimular los esfuerzos por garantizar la calidad, disminuyendo o suprimiendo por ejemplo la capacitación para las personas empleadas en el servicio o la reducción de mecanismos de supervisión y monitoreo de calidad y, por tanto, poniendo en jaque la efectiva protección del derecho a ser cuidado de las personas en situación de dependencia. De acuerdo con Geron et al (2000), el análisis de la calidad de los servicios, más allá de la presión por la reducción de los costos en el caso de los estados de bienestar, constituye una responsabilidad básica de aquellos que proveen cuidado a esta población y pretende garantizar derechos adquiridos. Asimismo, en América Latina, a partir de los noventas con el denominado Consenso de Washington y la introducción de las políticas neoliberales, la reducción de los gastos del Estado fue concebida como una necesidad para la mejora de la eficiencia y modernización de las instituciones públicas. En particular, las políticas de reducción del gasto social supusieron para muchos países la supresión y o tercerización de los servicios sociales. En esta línea, Fleury (1999) plantea que el papel que jugaron los Estados en America Latina no pudo contrarrestar las disparidades entre las regiones y sus ciudadanos teniendo al adoptar una lógica mercantil en la prestación y provisión de los servicios públicos.

En paralelo al interés en el tema de la calidad a nivel de los gobiernos con estados de bienestar consolidados en Europa surgido en el contexto de reducción o eficiencia de los gastos del Estado en materia social, varias investigaciones académicas se centraron en el estudio de la calidad de los servicios sociales y de cuidados, focalizándose en el análisis de cuáles son sus dimensiones centrales y potenciales indicadores. Así, es posible distinguir, en líneas gruesas, tres grandes perspectivas o enfoques. Por un lado, los en- 
foques que tienen en cuenta de manera exclusiva la dimensión subjetiva de la calidad, es decir, que se enfocan en el estudio de la percepción o valoración de las personas involucradas en el cuidado y, en particular, de las/los usuarias/os o beneficiarias/os del servicio en relación a su grado de satisfacción con la atención recibida. Una segunda perspectiva concibe que la calidad del cuidado está vinculada fundamentalmente con los aspectos materiales y organizacionales del proceso de producción técnica del servicio. Por último, visiones más integrales la entienden como el resultado de un proceso de producción de servicios que integra aspectos técnicos y organizacionales y que uno de sus indicadores o medidas principales es la percepción o valoración subjetiva de las personas involucradas en el servicio (y en particular la de las usuarias).

En la primera corriente pueden ubicarse investigaciones que han vinculado la calidad del servicio al grado de satisfacción de acuerdo a la percepción de los clientes, incluyendo la valoración de la adecuación del cuidado provisto en términos de su capacidad de responder o satisfacer a las necesidades de las personas usuarias del servicio. De manera más específica, Edelbalk et al (1995), encuentran, a partir de la realización de una investigación empírica, que las tres características más valoradas en relación a la calidad según los adultos mayores atendidos en sus hogares son la continuidad del personal, la adecuación del cuidado provisto a las necesidades y la buena relación con quien cuida. Para los investigadores, la continuidad del servicio de atención en domicilio, tanto en términos funcionales como de las personas que lo proveen parece ser una precondición necesaria para que la persona que cuida en domicilio y la persona adulta mayor construyan una relación de confianza en donde esta se sienta segura. Además, reduce el tiempo destinado a las negociaciones o discusiones sobre aquello que debe ser realizado en el hogar. Morrow-Howell et al. (2000) sostienen que la calidad depende de la percepción de todas las personas involucradas en la provisión de cuidado. Plantean que las percepciones dependen de las expectativas y el valor dado a los distintos aspectos del cuidado, así como de las posiciones que se ocupen en el proceso de producción del cuidado (personas cuidadas y sus familiares, proveedores de cuidados, cuidadores-as, supervisores, administradores, responsables y reguladores del servicio).

En la segunda perspectiva se presentan investigaciones centradas en los procesos de producción de los servicios en tanto aspecto que explica, en parte, el grado de satisfacción del cliente o calidad del servicio. (Freidson, 1961). Para Greenley y Schoenherr (1981). La satisfacción con respecto al servicio es resultado de las características organizacionales, siendo la calidad concebida como un asunto de efectividad organizacional (Price 1972), en el que se destacan aspectos tales como la estructura de organización interna y los procesos del proveedor del servicio que inciden en el desempeño organizacional.

En el tercer enfoque podrían situarse a Malley y Fernández (2010), quienes sostienen que la calidad del cuidado social presenta distintos desafios conceptuales y analíticos, no sólo por su 
compleja multidimensionalidad en cuanto a elementos técnicos ligados al funcionamiento del servicio, sino también por los diferentes significados dados a la calidad por quienes se encuentran involucrados en el cuidado. Las dimensiones o aspectos estructurales del servicio tienen que ver con las características relativamente estables de los proveedores de cuidado tales como las herramientas y recursos que tienen a su disposición y las configuraciones fisicas y organizacionales dentro de las cuales se trabaja (Donabedian, 1980).

Desde nuestro punto de vista, la valoración de la calidad de los cuidados provistos en el domicilio varía de acuerdo con las distintas percepciones de las personas involucradas (personas atendidas, familiares, responsables y-o proveedores del servicio). Dichas valoraciones y, en particular, la de las personas usuarias del servicio, constituyen uno de los indicadores subjetivos más relevantes a la hora de conocer cuál es la calidad de los servicios en términos del grado de satisfacción con la atención provista. No obstante, aquello que explica en gran medida la percepción sobre la calidad de la atención recibida depende de la forma en que el proceso de producción del servicio o atención recibida es realizada. Cuando la atención en domicilio es llevada a cabo en el marco de una prestación o servicio de responsabilidad estatal, las condiciones técnicas, materiales, organizacionales que la enmarcan, inciden en la forma en la cual el cuidado es brindado a las personas usuarias. Por tanto, si bien la calidad de un servicio es susceptible de ser medida mediante indicadores subjetivos como la percepción de quienes están involucrados en la provisión del mismo, los aspectos técnicos, materiales y organizacionales que forman parte del contexto en el que la atención es provista condicionan la forma en que los cuidados son provistos. Estos aspectos afectan a la calidad de la atención brindada a las personas en situación de dependencia en sus domicilios, lo cual impacta en la efectiva protección del derecho a ser cuidados. ${ }^{1}$ Entre los aspectos más destacados pueden señalarse la adecuada y suficiente formación específica en cuidados en tanto requisito para acceder al trabajo de cuidados en domicilio, así como la formación permanente en el puesto de trabajo, el soporte técnico con el que quienes brindan la atención cuentan a nivel institucional y organizacional -tanto para la resolución de emergencias como para el seguimiento y monitoreo de casos-, así como la existencia de dispositivos organizacionales orientados a prevenir los riesgos laborales (reuniones presenciales de coordinación individual y grupal, entre otros)- y las condiciones de trabajo (salario, modalidad de contrato, posibilidades de promoción o ascenso laboral). Los apoyos o soportes técnicos con los que cuenten las personas que brindan la atención directa, son claves para alivianar el peso de la responsabilidad y los riesgos laborales específicos derivados del trabajo de cuidados, el cual es vivido en la soledad y aislamiento respecto de otros trabajadores en la medida en que es llevado a cabo en la intimidad de los hogares de las personas usuarias del servicio.

En suma, nos posicionamos desde el enfoque holístico o integrador de la calidad anteriormente presentado, al considerar que esta tiene que ver, y de hecho es susceptible de 
ser medida a partir de la valoración subjetiva de los actores involucrados en su proceso de provisión, que es también resultado de aspectos y condiciones técnicas, materiales y organizacionales que pautan la forma en la cual la atención en domicilio es brindada.

\section{La calidad del cuidado provisto en el marco del Programa de Asistentes Personales del Sistema Nacional Integrado de Cuidados del Uruguay}

En Uruguay la ley 19.353 creó el Sistema Nacional Integrado de Cuidados (SNIC), orientada a atender algunas de las necesidades de cuidado de las personas en situación de dependencia. ${ }^{2}$ El SNIC se gestó durante un largo tiempo de discusiones políticas y sociales que comenzaron en 2009 hasta que en 2015, luego de promulgada la ley, fue lanzado el primer Plan Nacional de Cuidados a ejecutarse desde 2016 hasta 2020. En el mismo se establecen los objetivos del sistema, las poblaciones objetivo con las que trabajará y qué acciones se prevén para el mencionado período. El Sistema se plantea como objetivo general

Garantizar el derecho de las personas en situación de dependencia a recibir cuidados en condiciones de calidad e igualdad, promoviendo el desarrollo de la autonomía, la atención y asistencia a las personas en situación de dependencia, así como el desarrollo infantil, en el marco de un modelo de corresponsabilidad entre familias, Estado, mercado y comunidad, así como entre varones y mujeres (SNIC, 2015:11). ${ }^{3}$

Entre las prestaciones ofrecidas por el sistema o política pública específica de cuidados se encuentra el programa de Asistentes Personales (AP), consistente en una prestación económica para el pago de 20 horas de asistencia y cuidados en domicilio a ser provista por un Asistente Personal formado y habilitado por el propio programa. Dicho programa ya funcionaba en el período anterior (2010-2015) pero bajo la órbita del Banco de Previsión Social (BPS). En 2015 con la creación del SNIC, dentro del Ministerio de Desarrollo Social, el programa fue trasladado a esa dependencia institucional y reconfigurado, fundamentalmente a nivel de los requisitos para su acceso (antes no se evaluaba el nivel económico de las familias y personas dependientes que lo solicitaban) y de la oferta formativa en cuidados exigida para desempeñarse como asistentes personales en tanto requisito para ser registradas y habilitadas por el Programa, el cual previamente no era necesario para realizar trabajo de cuidados en domicilio. 
En la actualidad, el programa AP busca brindar apoyo a las personas en situación de dependencia severa, siendo la asistencia personal concebida como

el servicio que atiende el cuidado y la asistencia personal para las actividades de la vida diaria, es decir, que precisan ayuda para satisfacer las necesidades básicas de la vida diaria. ${ }^{4}$

El programa cuenta con dos tipos de componentes. Por un lado, una prestación monetaria para el pago de un/a Asistente Personal por unas horas determinadas por semana de atención en domicilio para personas en situación de dependencia categorizada como severa y, por el otro, un sistema de formación en cuidados básico para quienes deseen trabajar como Asistentes Personales y que una vez formados son registrados y habilitados por el Programa.

En relación con el primer componente, la prestación económica es otorgada a las familias o las propias personas en situación de dependencia severa menores de 29 años o mayores de 80 y cuyos ingresos no excedan once BPC $^{5}$ (36.740 pesos uruguayos per cápita de ingresos mensuales o mil doscientos de dólares aproximadamente, según valores a diciembre del 2017) para el pago de un-a Asistente Personal. La transferencia monetaria consiste en una liquidación y pago de 11.862 pesos uruguayos nominales (400 dólares aproximadamente) por 80 horas mensuales de trabajo. ${ }^{6}$ Para determinar el nivel de dependencia se utiliza el baremo de dependencia, un indicador que tiene en cuenta la autonomía de las personas para realizar por sí solas las actividades básicas de la vida diaria.

Respecto a los criterios de acceso relativos a la edad de las personas usuarias del Programa es preciso mencionar que la elección realizada deja fuera a una gran proporción de la población en situación de dependencia y, particularmente, a las personas mayores de 65 años a 80 años, para quienes el soporte de una tercera persona para realizar las actividades de la vida diaria suele ser frecuente. Si se consideran todos los niveles de dependencia de las personas de 65 años y más, la cobertura del programa AP se acerca a cubrir tan sólo el 4\% de la población que potencialmente precisaría el servicio, considerando sólo a las personas que tienen dificultades para hacer actividades de la vida diaria de manera permanente. ${ }^{7} \mathrm{Al}$ respecto, en atención a restricciones presupuestarias, se definió un financiamiento diferenciado según el nivel de ingresos per cápita y la carga de cuidados del hogar. En ese sentido, los hogares pueden recibir el 100\%, el 66\%, el $33 \%$ o 0\% del financiamiento del programa AP. ${ }^{8}$ Cabe mencionar que en 2016 el 73,6\% de los beneficiarios recibieron el 100\% de la financiación del programa AP. Por tanto, en la actualidad, el poder adquisitivo de los hogares es otro de los criterios elegidos para otorgar el subsidio económico para el pago de un Asistente Personal, siendo el copago progresivo con relación al ingreso del hogar. Como resultado de la actual focalización del programa, en 2016 se estimó una atención a 3500 personas con dependencia severa, en el marco del establecimiento de un régimen de copago. ${ }^{9}$ 
El segundo componente del Programa se aboca a la formación de Asistentes Personales, mediante la oferta de un curso específico en cuidados de entre 90 a 150 horas que habilita a quienes lo aprueban a desempeñarse como Asistentes Personales y a su inscripción en el registro creado a tales efectos. También es posible que la persona beneficiaria de la prestación proponga a alguien con quien ya tenga una relación de cuidados para formarse como Asistente Personal en el Programa. Ahora bien, la cuestión de si dicha formación es suficiente o adecuada para desempeñarse adecuadamente en el puesto de trabajo de acuerdo a las exigencias que el trabajo tiene es parte del análisis sobre la calidad de la atención provisto en el marco del Programa.

Tal como lo hemos señalado anteriormente, concebimos que la calidad del cuidado provisto y en este caso de aquel brindado en el marco del programa AP, depende en parte de las condiciones bajo las cuales la atención a las personas es llevada a cabo en sus domicilios. Sin embargo, para analizar las condiciones materiales, técnicas y organizacionales del trabajo de las Asistentes Personales y cómo ello impacta en la calidad de la atención provista, es preciso tener en cuenta las características del trabajo remunerado de cuidados de personas en situación de dependencia en domicilio. En este sentido, un aspecto que distingue a este trabajo de aquel llevado a cabo en instituciones es la soledad y alta responsabilidad que recae sobre quien cuida. La misma está acompañada de márgenes de autonomía elevados en donde la resolución de múltiples problemas que emergen en los hogares que deben ser asumidos por las y los trabajadoras y trabajadores en un contexto de aislamiento físico (Van Rompaey, 2015). El trabajo de cuidados en domicilio consta de dos dimensiones centrales que estructuran las prácticas y rutinas de trabajo: la física y la emocional. La primera implica brindar soporte físico para la realización de las actividades básicas de la vida diaria (vestirse, higienizarse, alimentarse, trasladarse, etc.) y es la cara más visible, regulada y explicitada del trabajo. La segunda, supone proveer de soporte emocional a las personas atendidas (escucharlas, animarlas, consolarlas, etc.) y requiere también de la gestión o regulación de las propias emociones de las trabajadoras tanto en el puesto de trabajo como fuera de él.

De ambas dimensiones señaladas devienen riesgos laborales específicos. Así, las tareas que las trabajadoras tienen que realizar para brindar soporte físico a las personas dependientes traen aparejadas problemáticas de salud entre las que suelen encontrarse los problemas de espalda, rodillas, cansancio, entre otras (Van Rompaey, 2015).Vinculada a la dimensión emocional del trabajo aparecen riesgos laborales ligados a la sobrecarga emocional inherente a la atención a personas en condiciones que suelen ser de gran vulnerabilidad física y emocional y la consecuente necesidad de resolver o brindar respuesta ante situaciones cotidianas y de emergencia de carácter previsible e imprevisible.

El trabajo de cuidados en domicilio requiere de una amplia formación específica que permita dotar a quienes lo desempeñen de herramientas tanto teóricas (sobre salud 
en general y enfermedades específicas en particular), como prácticas (técnicas de higiene, movilización, etc.) para realizar el trabajo de manera adecuada o que al menos les permita mitigar los riesgos laborales asociados al puesto de trabajo. Sin embargo, el programa AP exige la realización de un curso de 150 horas semanales aproximadamente para obtener la habilitación para el trabajo, siendo la exigencia del nivel educativo haber aprobado la educación primaria completa. En este sentido, cabe preguntarse si dicha formación es pertinente en materia de contenidos y suficiente en relación a su duración. En una investigación que recoge la valoración de la formación recibida por las trabajadoras en el Servicio Público de Atención en Domicilio del Ayuntamiento de Barcelona (Van Rompaey, 2015), se constata que las trabajadoras perciben que la formación recibida -teniendo en cuenta que el mínimo exigido para acceder al puesto de trabajo es de 350 horas- es insuficiente para poder desempeñarse adecuadamente, por la complejidad de las condiciones y situaciones de las personas atendidas, sus variadas problemáticas, enfermedades y comportamientos. En consecuencia parece sensato argumentar que, dado que las Asistentes Personales atienden a las personas con dependencia más severa, la formación ofrecida y exigida por el SNIC, que consta de como máximo 150 horas, resulte insuficiente en términos de su duración.

Respecto al soporte técnico y dispositivos de prevención de riesgos laborales que caracterizan al programa AP en su actualidad, las y los Asistentes Personales que realizan la atención directa en los hogares no cuentan con ningún tipo de soporte o apoyo técnico a nivel organizacional. En este sentido, las/los AP no cuentan con personal de apoyo que coordine su desempeño y actuación ni que sea referente para asistir técnicamente cuando se presentan casos o situaciones complejas con las personas atendidas, así como para la realización del monitoreo y seguimiento de los casos atendidos. Tampoco el Programa establece mecanismos de prevención de riesgos laborales para un trabajo complejo y marcado por la soledad y elevados grados de responsabilidad respecto al bienestar de las personas atendidas en los domicilios. No se contemplan mecanismos específicos permanentes de prevención de los riesgos laborales específicos tanto a nivel emocional como físico, lo cual implica una reproducción de las deficitarias condiciones que ya tienen las cuidadoras remuneradas tanto formales como informales en Uruguay. Los y las AP tampoco cuentan con un protocolo de actuación donde se expliciten las responsabilidades básicas, los derechos y responsabilidades derivadas de su perfil o rol profesional.

Por otra parte, el salario que se brinda a los Asistentes Personales es muy bajo, si se considera lo establecido como salario mínimo nacional (11.500 pesos uruguayos, según valores en 2016). De esta manera, el trabajo de cuidados es mercantilizado por el propio Estado como un trabajo de los peores pagos. Esto refuerza un fenómeno que ya sucede con el trabajo doméstico y de las cuidadoras en domicilio remuneradas Uruguay, representándolo como un trabajo de escaso valor, en vez de contribuir a que los salarios aumenten respecto por ejemplo al trabajo doméstico y a que los cuidados se valoren como empleo y una profesión. 
La contratación de Asistente Personal se lleva a cabo por los propios usuarios o a través de sus representantes, pudiéndose establecer el contrato con una persona física o jurídica (ley 19.353, art. 22). El o la Asistente Personales debe informar al Banco de Previsión Social (BPS) la cantidad de horas trabajadas, independientemente de si los hogares reciben el subsidio económico no. Actualmente en el vínculo funcional con el BPS, los y las Asistentes Personales constituyen un grupo específico, expresado en la codificación 115. Se prevé en el contrato y el subsidio brindado por el SNIC el pago de salario vacacional y de aguinaldo, aunque no queda claro el tiempo de contratación y la posterior seguridad laboral de los Asistentes Personales una vez que terminan el vínculo laboral con el beneficiario o su representante. Si bien el tipo de contrato está asociado a la habilitación por parte del sistema y genera garantías de salario vacacional y licencia, la estabilidad del empleo depende en mayor medida de los hogares contratantes. En este sentido, se asemeja al trabajo doméstico, siendo la precariedad laboral una característica que afecta a los y las AP como trabajadores y trabajadoras.

En relación a la cuestión del grado de satisfacción de las personas usuarias del programa, así como del resto de actores involucrados en el proceso de provisión de cuidados, el SNIC no cuenta con información disponible en la actualidad, razón por la cual no disponemos de indicadores que permitan conocer la calidad del cuidado en domicilio de acuerdo con la valoración subjetiva.

En síntesis, desde el punto de vista de sus condiciones materiales y técnicas, dada la ausencia de soporte técnico para quienes llevan a cabo la atención en domicilio, mecanismos organizacionales de prevención de riesgos laborales, la baja carga horaria de la formación de las trabajadoras y las malas condiciones laborales, a la par que la inexistencia de un sistema de evaluación del grado de satisfacción respecto al cuidado provisto, van en detrimento de la medición de la calidad actual y de las posibilidades de mejora de la atención brindada.

\section{Reflexiones finales}

Una de las principales reflexiones que surgen del análisis del actual programa Asistentes Personales de Uruguay en relación a las condiciones técnicas bajo las cuales la atención en domicilio es provista radica en que no existe soporte técnico durante la actividad de cuidados en domicilio, ni seguimiento desde el programa de los casos atendidos; tampoco existen mecanismos de prevención de riesgos laborales (tanto físicos como emocionales). A su vez, las condiciones laborales o materiales que enmarcan el trabajo desarrollado por quienes brindan atención en domicilio, no sólo se caracterizan por la baja remuneración percibida y una modalidad contractual precaria, sino también por una baja carga horaria de la formación requerida para el acceso de los y las asistentes personales al Programa. En este sentido, sería importante contar con información relativa a la valoración por parte de las Asistentes Personales sobre de la formación específica recibida, su suficiencia, así como la pertinencia de los contenidos. 
El Programa no constituye en la actualidad un servicio -en el sentido de que cuente con los dispositivos y mecanismos organizacionales de soporte técnico y prevención de riesgos laborales-, capaz de reflejar un Estado responsable por el seguimiento y monitoreo del cuidado provisto y de la situación de las personas usuarias del programa.

La ausencia de información sobre la valoración del programa por parte de las personas usuarias dificulta conocer cuál es el grado de satisfacción respecto a la calidad de la atención brindada. Sumado a que la prestación económica no cubre a una gran porción de personas que, en situación de dependencia, requieren de soporte para llevar adelantes diariamente sus vidas en sus hogares, estos aspectos cuestionan la capacidad real del Estado uruguayo para garantizar el derecho a ser cuidado. A más de dos años de la existencia del SNIC, sólo una población focalizada por edad, grado de dependencia e ingresos económicos puede acceder a la prestación monetaria del programa AP. Ello pone en tela de juicio el pleno goce del derecho a ser cuidado de las personas en situación de dependencia, en la medida en que la prestación no es de carácter universal, tal como fue señalado porVan Rompaey y Scavino (2018).

Por otro lado, generar sistemas de monitoreo y evaluación de la calidad de la atención provista por parte de las personas usuarias y sus familias, así como mejorar de las condiciones técnicas y laborales bajo las cuales la atención directa es actualmente realizada por los y las Asistentes Personales en el Programa AP del Uruguay, son aspectos que no sólo permitirían conocer cómo es valorado el cuidado actualmente recibido, sino que probablemente permitan mejorar la calidad de atención y garantizar de manera más efectiva la protección de derecho a ser cuidado de la población en situación de dependencia.

Adhiriendo a una perspectiva holística de la calidad, consideramos que no basta con conocer cuál es la percepción de las personas usuarias del programa AP, sino que el Estado asuma su responsabilidad última en la producción global de la atención provista en los domicilios de las personas en situación de dependencia. La atención directa llevada a cabo por las y los Asistentes Personales se enmarca en ciertas condiciones materiales y técnicas que afectan la calidad del cuidado provisto, más allá de la voluntad de las cuidadoras y su vocación de servicio. Es por ello que el desafio de garantizar el efectivo ejercicio del derecho a ser cuidado de las personas en situación de dependencia en el caso uruguayo requiere de un mayor compromiso estatal no sólo para lograr el acceso universal de la población a la prestación económica, sino también de responsabilizarse por el proceso de producción de la atención en domicilio y la calidad de los cuidados provistos en este ámbito. En esta línea, avanzar en la creación de condiciones materiales y técnicas donde quienes llevan a cabo la atención directa cuenten con soporte técnico (tanto para la resolución de emergencias, como para el seguimiento de los casos), así como con el respaldo de mecanismos de prevención de riesgos laborales, en el marco de mejores condiciones de trabajo, contribuirían a reforzar la garantía de la real protección estatal del derecho a recibir cuidados, -al menos en los domicilios-, de las personas en situación de dependencia. 


\footnotetext{
${ }^{1}$ Autoras pioneras en plantear la cuestión de la calidad de los cuidados públicos y su vinculación con la efectiva protección del derecho a ser cuidados en el marco de la concepción de una ciudadanía inclusiva fueron Knijn y Kremer (1997). Al respecto consideran que el Estado tiene que asumir o no la responsabilidad del cuidado y asegurar a los ciudadanos el derecho a dar y recibir cuidado, pero además resolver qué medida, a qué costo y sobre la base de qué tipo de supuestos y condiciones es dicha responsabilidad asumida. Así, las condiciones bajo las cuales el cuidado es brindado y desarrollado son decisivas en su calidad y por ende están vinculadas a la garantía de que los derechos puedan ser ejercidos y respetados.

${ }^{2}$ La dependencia es definida en la Ley 19.353 como "el estado en el que se encuentran las personas que requieren de la atención de otra u otras personas o ayudas importantes para realizar las actividades básicas y satisfacer necesidades de la vida diaria". Disponible en http://www.sistemadecuidados.gub.uy/innovaportal/file/58642/1/ley-19.353---sistema-de-cuidados.pdf.

${ }^{3}$ Entre los objetivos específicos se incluyen el aumento de la cobertura y la mejora de la calidad de servicios para la primera infancia, mejorar la atención de personas mayores y con discapacidad en situación de dependencia, el fortalecimiento de capacidades institucionales y su mejora continua, la constitución de un marco legal para que se reconozca el derecho a cuidar y ser cuidado en condiciones de calidad, el desarrollo de oferta de formación para el trabajo de cuidados, la producción y gestión de la información oportuna y pertinentes sobre los sujetos de la política y las prestaciones y efectos del SNIC, la generalización del reconocimiento público del derecho a los cuidados mediante una estrategia comunicacional que contribuya a generar una transformación cultural para un mayor compromiso de corresponsabilidad social en los cuidados (SNIC, 2015: 11). ${ }^{4}$ http://www.sistemadecuidados.gub.uy/innovaportal/v/61052/1/innova.front/asistentes-personales

${ }^{5}$ BPC es la sigla de "Base de Prestaciones y Contribuciones". Es una unidad de referencia creada por el artículo 2 de la Ley $N^{\circ} 17.856$ y vigente desde 2005 utilizada para sustituir a todas las referencias al salario mínimo nacional (SMN) establecidas en el ordenamiento jurídico vigente.

${ }^{6}$ http://www.mides.gub.uy/innovaportal/v/63068/3/innova.front/es-hoy

${ }^{7}$ Estimaciones realizadas en base a la población dependiente detectada por el baremo en la ELPS (2013) y los datos de cobertura del programa de AP (Scavino, 2017).

${ }^{8}$ http://www.sistemadecuidados.gub.uy/84284/informe-anual---cuidados-rinde-cuentas.

${ }^{9}$ http://www.mides.gub.uy/innovaportal/v/63068/3/innova.front/es-hoy
} 


\section{Referencias bibliográficas}

Applebaum, R. y Phillips, M. (1990): “Assuring the Quality of In-Home Care:The Other Challenge for Long-Term Care”. Gerontologist, No. 30,Vol. 4. 444-450.

Bazo, María Teresa (1998): "Vejez dependiente, políticas y calidad de vida". Papers, No. 56. 143-161.

Belt, B.; Richardson, R. y Webster,J. (2002): "Women, social skills and interactive service work in telephone call centres”. New Technology, Work and Employment, No. 1,Vol. 17. 20-34.

Donabedian, A. (1980): The definition of quality and approaches to its assessment. Ann Arbor, MI: Health Administration Press.

Durán, María de los Ángeles (2006): "Dependientes y cuidadores: el desafio de los próximos años”. Revista del Ministerio de Trabajo y Asuntos Sociales, No. 60. 57-71.

Edelbalk, G., Samulesson, G. y Ingvad, B. (1995): "How elderly people rank the quality characteristics of home services”. Ageing and Society, No. 1,Vol.15. 83-102.

Escudero, B. (2007): "Las políticas sociales de dependencia en España”. Revista Española de Investigaciones Sociológicas, No. 119,Vol. 1. 65-89.

Fleury, S. (1999): “Reforma del estado en América Latina ¿Hacia dónde?” Nueva Sociedad, No. 160. 58-80.

Freidson, E. (1961): “The organization of medical practice and patient behavior." American Journal of Public Health and the Nations Health, No. 51,Vol. 1. 43-52.

Geron, S. M., Smith, K., Tennstedt, S., Jette, A., Chassler, D. y Kasten, L. (2000): "The home care satisfaction measure: a client-centered approach to assessing the satisfaction of frail older adults with home care services." The Journals of Gerontology Series B: Psychological Sciences and Social Sciences, No. 55,Vol. 5. 259-270.

Greenley, J. R., y Schoenherr, R. A. (1981): “Organization effects on client satisfaction with humaneness of service." Journal of Health and Social Behavior. No.2.Vol.1.2-18.

Hampson, I. y Juno, A. (2005): “Invisible work, invisible skills: interactive customer service as articulation work". New Technology, Work and Employment, No. 2,Vol. 20.166-181. 
Knijn, T. y Kremer, M. (1997): “Gender and the Caring Dimension of Welfare States: Toward Inclusive Citizenship”. Social Politics, No. 4,Vol. 3. 328-361.

Korczynski, M. (2002): Human Resource Management in Service Work. Londres-Nueva York: Palgrave Macmillan.

Korczynski, M. y Macdonald, C. (2009): Service work. Critical perspectives. Nueva York: Routledge.

Leichsenring, K. (2004): "Developing integrated health and social care services for older persons in Europe.” International Journal of Integrated Care, No. 3. None.

Lloyd-Williams, M. y Payne, S. (2002): "Can multidisciplinary guidelines improve the palliation of symptoms in the terminal phase of dementia?.” International Journal of Palliative Nursing, No. 8, Vol. 8. 370-375.

López Casasnovas, G. (2011): “La Ley de autonomía y dependencia. Reflexiones y recomendaciones para su mejor desarrollo”. Gaceta sanitaria, No. 25. 125-130.

Malley, J. y Fernández, J. L. (2010): “Measuring quality in social care services: theory and practice". The Annals of Public and Cooperative Economics, No. 81, Vol. 4. 559-582.

Morrow-Howell, N. et al (2000): "How Much Is Enough? Perspectives of Care Recipients and Professionals on the Sufficiency of In-Home Care". The Gerontologist, No. 6,Vol.11.143-161.

Nuñez, I. (2014): "Personas con discapacidad en Uruguay: algunas cifras del Censo 2011." Disponible en: https://www.bps.gub.uy/bps/file/8195/1/44._personas_con_ discapacidad_en_uruguay._algunas_cifras_del_censo_2011._nunez.pdf

Jiménez Lara, A. y Huete García, A. (2009): “Políticas públicas sobre discapacidad en España. Hacia una perspectiva basada en los derechos." Política y Sociedad, No. 47,Vol. 1. 137-153.

Payne, J. (2009): “Emotional Labour and Skill: A Reapraissal.” Gender, Work and Organization, No. 3,Vol. 16.348-367.

Price, J. L. (1972): “Handbook of organizational measurement." International Journal of Manpower. No. 18,Vol. 4. 305-558.

Rigby, M. y Sanchis, E. (2006): "El concepto de cualificación y su construcción social”. Revista Europea de Formación Profesional. No. 37. 24-35. 
Rodríguez Cabrero, G. (2007): “La protección social de la dependencia en España. Un modelo sui generis de desarrollo de los derechos sociales". Política y sociedad, No. 44,Vol., 2. 24-35.

Scavino, S. (2017): “Familismo soportado y feminización de las estrategias de cuidado en salud. Desafios persistentes para el derecho al cuidado y la equidad de género.” Tesis de Maestría en Sociología. Universidad de la República, Montevideo, Uruguay.

Senlles, A. y Gutiérrez, N. (2005): Calidad en los servicios educativos. Díaz de Santos.

Thompson, P.;Warhurst, C. y Callaghan, G. (2001): "Ignorant theory and knowledgeable workers: Interrogating the connections between knowledge, skills and services." Journal of Management Studies, No. 38, Vol. 7. 923-942.

Torres Pérez, L.A. (2005): Libro Blanco de la Dependencia en España. Disponible en http:// www.empleo.gob.es/es/publica/pub_electronicas/destacadas/Revista/numeros/60/Est03.pdf

Van Rompaey, E. (2015): “Cualificación invisibilizada. Estudio sobre el trabajo remunerado de cuidados en domicilio en los servicios de atención barcelonés y montevideano.” Tesis Doctoral sin publicar. Departamento de Sociología, Universidad Autónoma de Barcelona, Barcelona. España.

Van Rompaey, E. y S. Scavino, S. (2018): "Hacia una revalorización del trabajo de cuidados en domicilio.” Revista Punto y Género. No. 9 (en prensa).

Vehmas, S. y Watson, N. (2014): "Moral wrongs, disadvantages, and disability: a critique of critical disability studies.” Disability \& Society, No. 4,Vol. 19. 638-650.

Verdugo, M. A. (2003): La concepción de discapacidad en los modelos sociales. Investigación, innovación y cambio, Amaru, Salamanca.

\section{Cómo citar este artículo:}

Van Rompaey, Erika (2018) “La calidad del cuidado como desafio de las políticas públicas de atención a la dependencia”. Revista Perspectivas de Políticas Públicas vol. 8 №15: 89-106 